\title{
POSSIBILITY OF NEW LYMPHATIC PATHWAY CREATION THROUGH NEO-LYMPHANGIOGENESIS INDUCED BY SUBDERMAL DISSECTION
}

\author{
T. Yamamoto, N. Yamamoto
}

Department of Plastic and Reconstructive Surgery (TY,NY), National Center for Global Health and Medicine, Tokyo, Japan; Department of Plastic Surgery (TY,NY), Tokyo Metropolitan Bokutoh Hospital, Tokyo, Japan, Department of Plastic Surgery (TY), Noda Hospital, Chiba, Japan

\section{ABSTRACT}

Surgical intervention and subsequent wound healing process are known to induce neo-lymphangiogenesis, but few studies have been reported to utilize this mechanism for lymphedema treatment. The aim of this study was to evaluate feasibility of subdermal dissection for neo-lymphangiogenesis induction (SDN) to treat lower extremity lymphedema (LEL). Medical records of secondary LEL patients who had undergone ICG lymphography and SDN procedure were reviewed. SDN was performed by dissecting fat tissues just below the dermis from the most proximal area showing dermal backflow through abdominal-toaxillary lymphatic pathways. Perioperative lymphedematous conditions were evaluated with lymphedema quality of life score (LeQOLiS) and LEL index. Seventeen female patients were included. SDN could be performed in 10 minutes on average without postoperative complication. Postoperative ICG lymphography showed new lymphatic pathways in 6 (35.3\%) cases. Postoperative LeQOLiS ranged from 9 to 66, which was statistically lower than preoperative LeQOLiS (32.9 \pm 19.2 vs. $36.6 \pm 19.3, p=$ 0.048 ), whereas there was no statistically significant difference between pre- and post-operative $L E L$ index $(275.2 \pm 23.3$ vs. $270.5 \pm 20.8$, $P=0.073)$. Subdermal dissection, although its probability is not high, has a potential to induce neo-lymphangiogenesis. Further studies are required to improve and demonstrate efficacy of the procedure for new lymphatic pathway creation.

Keywords: lymphedema, manual lymph drainage, indocyanine green, lymphography, lymph flow, lymphangiogenesis, subdermal dissection

Lymphedema is a progressive edematous disease caused by abnormal lymph circulation (1-3). Lower extremity lymphedema (LEL) significantly affects patient's quality of life (QOL) and can occur due to primary without known cause or secondary cause o parasite infection, cancer treatment, trauma, or other events (1,2, 4-6). Conservative treatments such as skin care, compression therapy, manual lymph drainage (MLD), and exercise therapy are mainstays of lymphedema management $(1,4,6)$. However, they are non-curative and anti-symptomatic treatments, and most secondary LEL cases require life-long conservative treatments including compression therapy $(2,6-9)$. Various lymphedema operations have been utilized in progressive LEL cases refractory to conservative treatments and include lymphovenous anastomosis (LVA), lymph node transfer (LNT), and debulking surgery 
such as liposuction (9-15). Among them, LVA and LNT are used to improve lymph circulation.

LVA is indicated for mild-to-moderate lymphedema cases without severe lymphosclerosis (9-11, 16-19). Since LVA aims to move congested lymph into venous circulation via an anastomosis, a lymph vessel with lymph flow inside the lumen is required to expect therapeutic efficacy $(9,10,16,19)$. Efficacy of LVA would be minimal if a severely sclerotic lymph vessel is used for anastomosis $(11,16$, 20,21). Although this is a minimally invasive lymphedema surgery which can be done under local infiltration anesthesia, indication of LVA is limited to relatively early-stage lymphedema cases $(10,11,21)$. For progressed LEL cases with severe lymphosclerosis, LNT is applied $(10,12,13,20,22)$. Unlike LVA, LNT utilizes an intact lymphatic tissue and has a therapeutic efficacy even for severe LEL cases $(13,16,20$ 22). However, LNT requires general anesthesia, and has a risk of donor site lymphedema $(11,13,20,21,23,24)$. Therefore, indication of LNT should be carefully considered, and a better method is warranted for severe LEL cases refractory to LVA.

Several studies have reported the possibility of neo-lymphangiogenesis after surgical intervention $(20,21,25-27)$. Neo-lymphangiogenesis can be seen in conventional wound healing processes and is considered of potential to improve lymph circulation without LVA or LNT. New lymphatic pathways can be created via neo-lymphangiogenesis, which can facilitate efficacy of $\operatorname{MLD}(20,25,27)$. This study aimed to evaluate effect of a simple surgical intervention, subdermal dissection, on new lymphatic pathway creation via neo-lymphangiogenesis in secondary LEL cases.

\section{MATERIALS AND METHODS}

Medical charts of secondary LEL patients who had undergone ICG lymphography and subdermal dissection for induction of neo-lymphangiogenesis (SDN) were reviewed. This retrospective observational study was approved by institutions' ethical review boards, and all patients gave written informed consent to the study. Patients with a followup period shorter than 12 months were excluded. Clinical charts were reviewed to collect clinical and lymphographic findings. All patients were refractory to conservative treatments including MLD and LVA surgery. They also showed no collateral pathways from the lower extremity to the axilla on indocyanine green (ICG) lymphography (no Linear pattern in the anterior or lateral abdomen on ICG lymphography after ICG injection in the second web space of the foot as previously reported) (28-32).

SDN was performed under local infiltration anesthesia in a supine position as a day surgery without hospitalization. Preoperative ICG lymphography was performed 2 hours before SDN surgery to evaluate pathophysiological severity and to confirm extension of lower extremity dermal backflow and lack of abdominal-to-axillary (ATA) lymphatic pathways. For ICG imaging, $0.1 \mathrm{ml}$ of $0.25 \%$ ICG was intra-dermally injected at the second webspace of the foot and at the anterior and lateral abdomen at the level of the umbilicus. Based on ICG lymphography findings, extension of dermal backflow area and proposed ATA lymphatic pathways were marked with a pen. To bridge the gap between the proximal margin of dermal backflow area and the initial points of the ATA lymphatic pathways, multiple lines were designed and marked on the skin. Along the designed lines, $2-3 \mathrm{~cm}$ skin incisions were designed vertically to the lines for subdermal dissection (Fig. 1). After local infiltration anesthesia along the designed lines with $0.5 \%$ lidocaine with 1:100,000 epinephrine, skin incisions were made using a surgical scalpel. Via the skin incisions, subdermal tissues were dissected just below the dermis along the designed lines using Kelly forceps (Fig. 2). After the subdermal dissection, wounds were irrigated with saline, and hemostasis was confirmed. Wounds were closed with interrupted dermal stitches using 4-0 PDS and compressed with gauzes. The same compression garments as preoperatively used were resumed immediately after SDN surgery. One week after the SDN surgery, modified self MLD was introduced. The self MLD was performed 3 times a day along the designed lines 


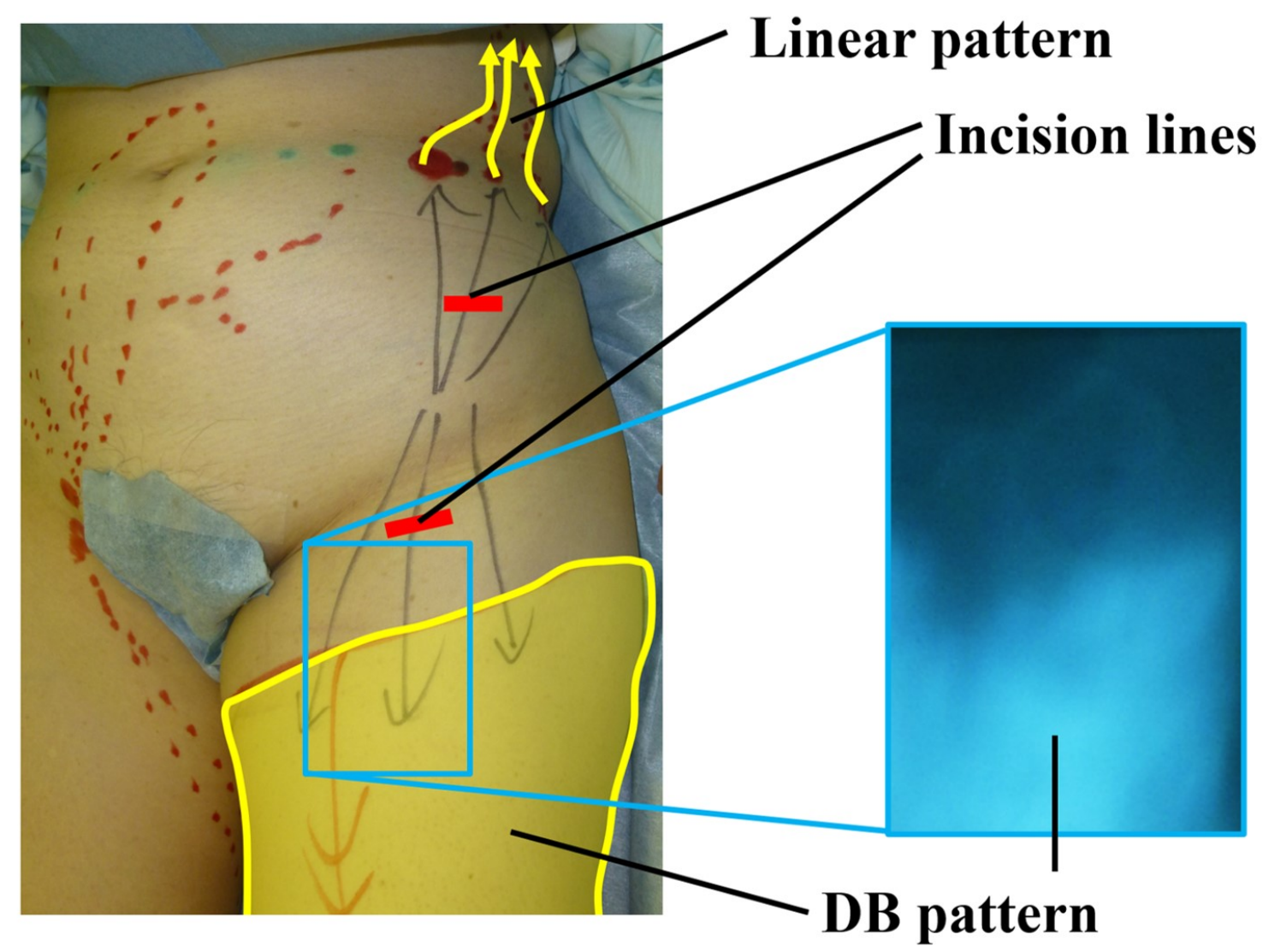

Fig. 1. Skin incision design for SDN procedure. Highlighted (yellow) segment on the leg represents area showing dermal backflow (DB) pattern (right), and arrows on trunk Linear pattern on ICG lymphography (not shown).

where subdermal dissection was performed in the SDN surgery (Fig. 3).

Patient characteristics, lymphedema history, physical findings, lymphographic findings, and operative findings were collected and evaluated. Lymphedematous conditions were evaluated with LEL index and lymphedema quality of life score (LeQOLiS) $(4,5)$. LEL index is body type-corrected lymphedematous volume evaluation method and is calculated using measurements of circumferences and body mass index (BMI) according to the following formula: $\mathrm{LEL}$ index $=(\mathrm{C} 12+$ $\mathrm{C} 22+\mathrm{C} 32+\mathrm{C} 42+\mathrm{C} 52) / \mathrm{BMI}(33,34) \cdot \mathrm{C} 1-5$ denote circumferences $10 \mathrm{~cm}$ above the superior border of the patella, at the superior border of the patella, $10 \mathrm{~cm}$ below the inferior border of the patella, at the ankle, and at the foot. LeQOLiS consists of 10 questionnaires regarding subjective lymphedematous symptoms (Table 1). A summation of each score ( 0 , least severe; 100, most severe) was used to evaluate perioperative lymphedema symptoms (4). One year after SDN operation, postoperative ICG lymphography was performed to evaluate development of new lymphatic pathways by SDN procedure. Creation of new lymphatic pathway was evaluated as positive when ICG lymphography showed Linear pattern to the axilla through the dissected area. Plus-minus values represented means \pm standard deviations. Fisher exact probability test and Student's paired t-test were used for statistical analyses to compare clinical findings before and 12 months after SDN surgery. A pvalue $<0.05$ was set as statistical significance. 


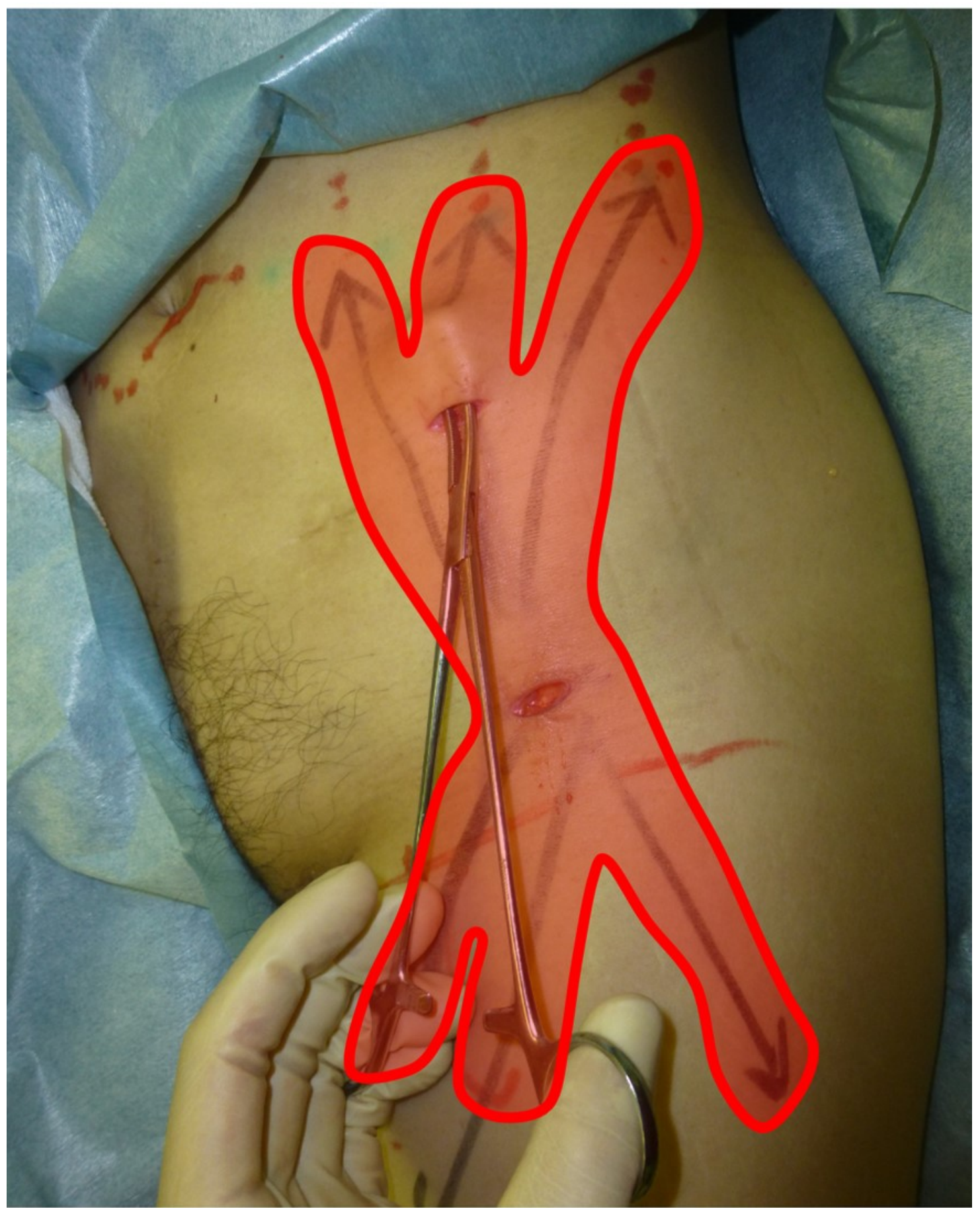

Fig. 2. Dissection area in SDN procedure. Fat tissues are dissected using Kelly forceps just below the dermis via 2 short skin incisions following the highlighted areas.

Permission granted for single print for individual use.

Reproduction not permitted without permission of Journal LYMPHOLOGY. 


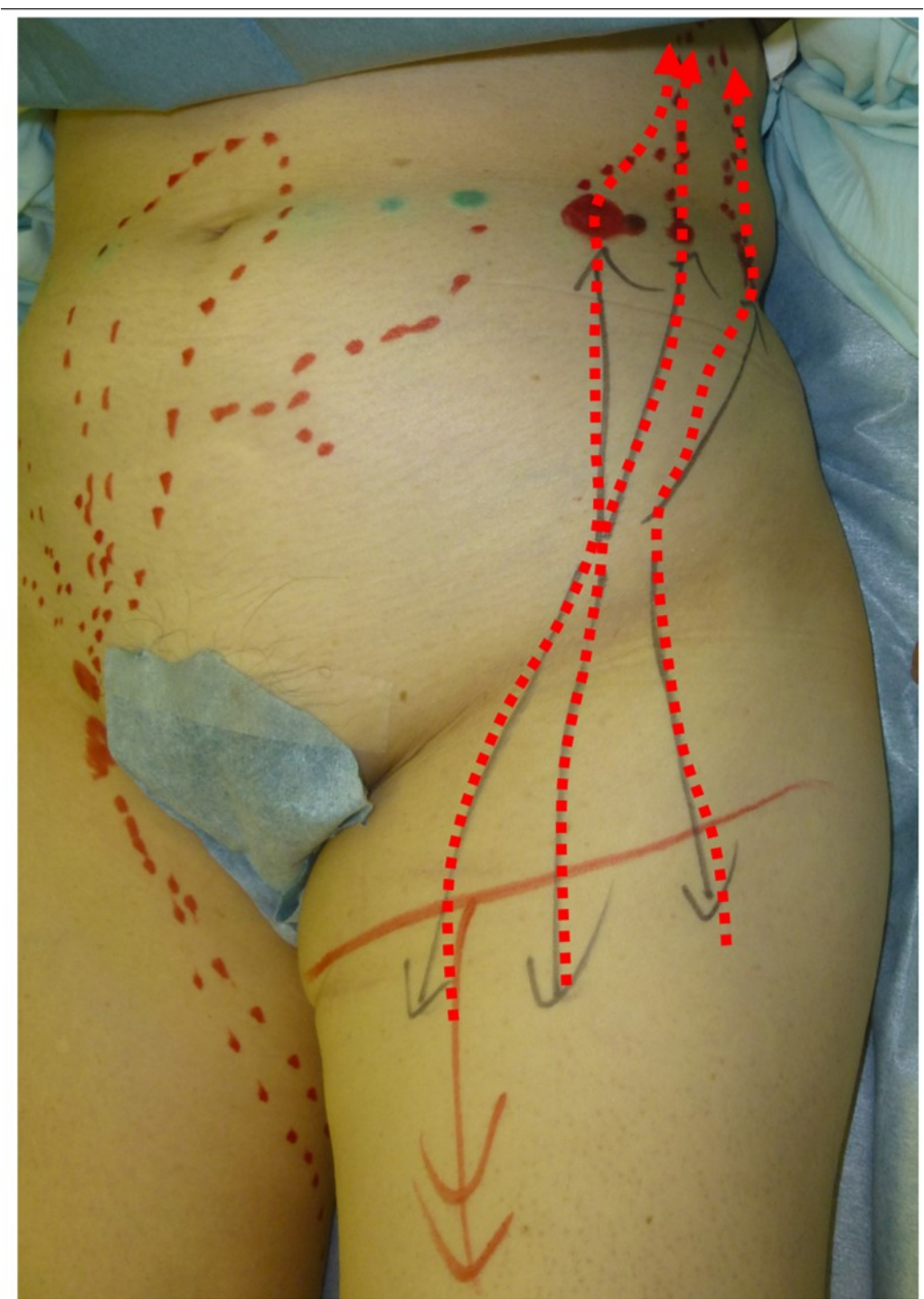

Fig. 3. Area and pattern for modified self-MLD. Dotted arrows represent massage directions in modified self-MLD from leg to trunk to encourage movement of fluid through the proposed new lymphatic pathways.

\section{RESULTS}

Seventeen limbs of 17 female patients were included in the study. Patient's age ranged from 41 to 86 years (average, 63.6 years). Patients' BMI ranged from 17.4 to $30.2 \mathrm{~kg} / \mathrm{m}^{2}$ (average, $\left.22.65 \mathrm{~kg} / \mathrm{m}^{2}\right)$. Etiology of lymphedema was secondary following cancer treatments in all cases; uterine cervical cancer in 9 (52.9\%), uterine corpus cancer in $6(35.3 \%)$, and ovari- an cancer in 2 (11.8\%). Duration of lymphedema ranged from 5 to 42 years (average, 18.4 years). Clinical stage of lymphedema included International Society of Lymphology stage 2 in 12 cases $(70.6 \%)$, and stage 3 in 5 cases (29.4\%) (8). Severity of lymphedema was ICG lymphography stage IV in 8 cases $(47.1 \%)$, and stage $V$ in 9 cases $(52.9 \%)(16,29)$. Complete decongestive therapy and LVA had been performed in all cases before SDN operation. 


\begin{tabular}{ll}
\multicolumn{1}{c}{ TABLE 1 } \\
\multicolumn{1}{c}{\begin{tabular}{c} 
TeQLiS Based on Questionnaires Regarding Lymphedema \\
\hline Questionnaire
\end{tabular}} \\
\hline Distension & $0-10 *$ \\
Heaviness & $0-10 *$ \\
Pain & $0-10 *$ \\
Dysesthesia & $0-10 *$ \\
Appearance Distortion & $0-10 *$ \\
Motor Dysfunction & $0-10 *$ \\
Limitations in Daily Activity & $0-10 *$ \\
Influence on Social Activity & $0-10 *$ \\
Distress by Conservative Treatment & $0-10 *$ \\
Overall Dissatisfaction caused by Lymphedema & $0-10 *$ \\
\hline LeQOLiS = Summation of scores described above & $0-100 * *$ \\
\hline
\end{tabular}

LeQOLiS, lymphedema quality of life score.

* 0 , least severe; 10 , most severe.

** 0 , least severe; 100 , most severe.

Preoperative LEL index ranged from 234 to 311 (average, 275.2), and LeQOLiS ranged from 11 to 71 (average, 36.6). Follow up period ranged from 12 to 28 months (average, 16.1 months).

Time required to complete SDN procedure ranged from 4 to $17 \mathrm{~min}$ (average, 9.1 min). All SDN procedures could be performed without any postoperative complication. New lymphatic pathways, which were not observed on preoperative ICG lymphography, were confirmed on postoperative ICG lymphography in $6(35.3 \%$ ) cases (Fig. 4). There was no statistically significant difference between postoperative and preoperative LEL indices $(270.5 \pm 20.8$ vs. $275.2 \pm 23.3, p=0.073)$. Postoperative LeQOLiS ranged from 9 to 66, which was statistically lower than preoperative LeQOLiS (32.9 \pm 19.2 vs. $36.6 \pm 19.3, \mathrm{p}=0.048)$.

\section{DISCUSSION}

This study revealed that SDN surgery improved QOL of patients with secondary
LEL refractory to conservative treatments and LVA. Although confirmed in only $35.3 \%$ of the cases on ICG lymphography, new lymphatic pathways were created by a simple surgical intervention of subdermal dissection without implanting any material or application of medical agent such as growth factor. To our knowledge, this is the first report of effect of subdermal dissection on new lymphatic pathway creation which was confirmed with ICG lymphography. Although not enough to induce in all cases, a simple surgical intervention of subdermal dissection has a potential to induce neo-lymphangiogenesis. This study also indicated that created new lymphatic pathways could be used to facilitate modified MLD. Previous studies reported that a site of surgical intervention showed a sign of neolymphangiogenesis (20,25-27). Most studies employed lymphoscintigraphy as an evaluation tool to detect postoperative lymph flow changes as it is considered a gold standard of lymph flow evaluation $(8,13,27)$. Although lymphoscintigraphy is usually performed to 


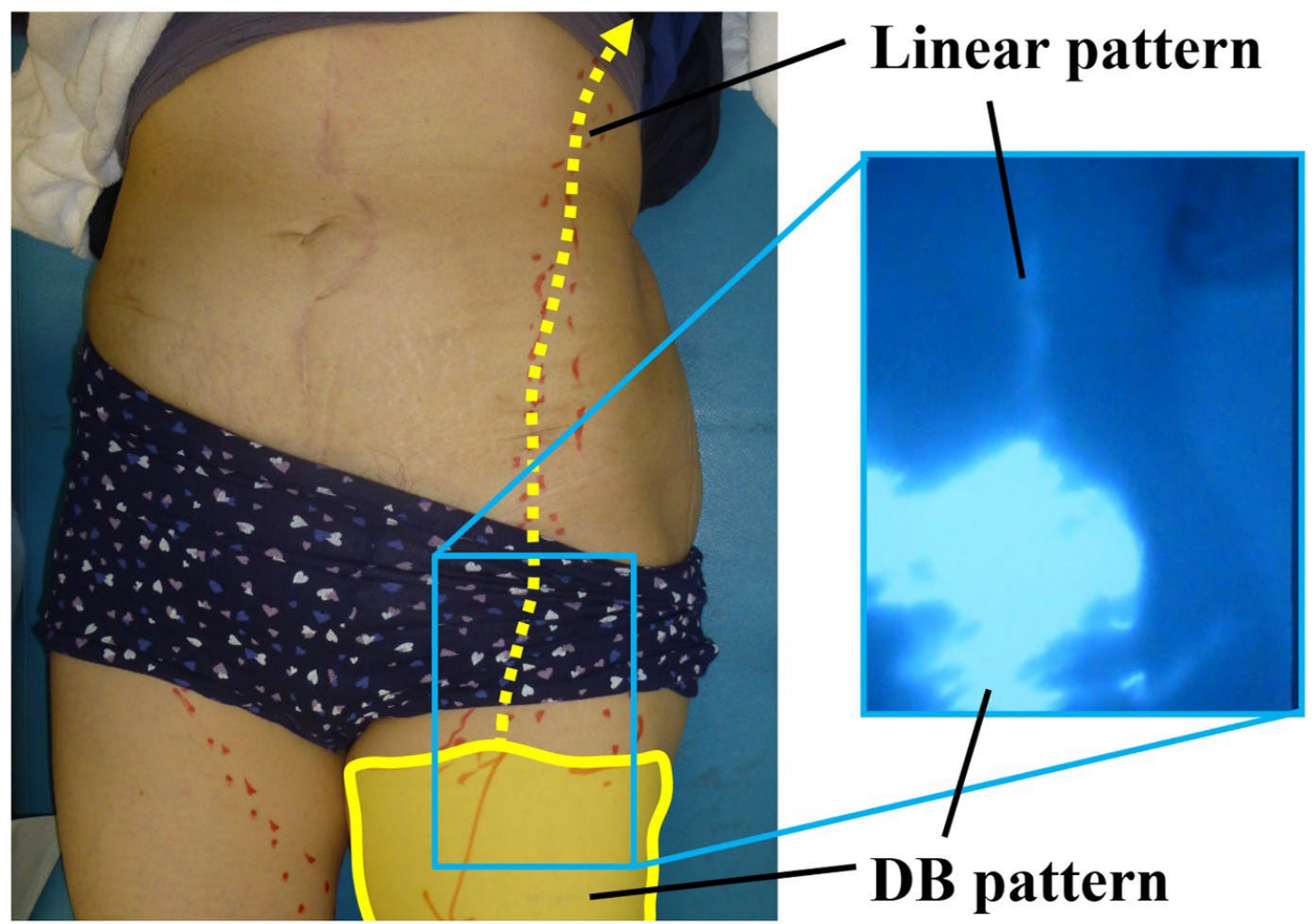

Fig. 4. Demonstration of new lymphatic pathway created after SDN procedure shown with ICG lymphography. Highlighted area on the leg depicts area showing dermal backflow (DB) pattern that has been reduced from the pre-operation image in Fig. 1 and development of a new Linear pattern by ICG which follows the dotted (yellow) arrow on the skin.

evaluate lymph circulation, creation of new lymphatic pathways should be evaluated using ICG lymphography, which has higher sensitivity and specificity to detect superficial lymph flow changes $(3,16,19,28,35)$. As tissues just below the dermis were dissected in this study, ICG lymphography was considered the best modality to detect superficial lymph flow creation $(19,31,36)$. Absence of new enhancement on ICG lymphography in 11 cases $(64.7 \%)$ suggested no new lymphatic pathway were created by subdermal dissection or the pathways did not functionally connect. Therefore, induction rate of neo-lymphangiogenesis by subdermal dissection is considered low. Nevertheless, the study results are of clinical significance that SDN has a potential to facilitate
MLD. As the procedures are quite simple and minimally invasive, SDN would be considered an acceptable option for progressed LEL cases refractory to LVA and not suitable for LNT, if further studies clarified its effectiveness.

Procedures applied in the study were simple and easy to perform in which only skin incision and subdermal dissection were performed with average time for procedure of 9.1 min. Although SDN surgery is simple and convenient, better procedures can be applied to induce neo-lymphangiogenesis. Skin incision alone may induce neo-lymphangiogenesis, but long skin incision is required to create new lymphatic pathways useful in MLD, leaving a long scar $(20,25)$. Liposuction can be done similarly to SDN in removing the fat tis- 
sues just below the dermis. As a liposuction canula is smaller than Kelly forceps, skin incision length would be shorter in liposuction method $(14,15,21,37)$. A major concern is success rate of neo-lymphangiogenesis induction. To improve the success rate, combination of other procedures may be useful. Previous studies reported neo-lymphangiogenesis induction by implanting artificial materials or by applying pharmaceutical agents $(26,27)$. However, artificial material implantation has a long-term risk of infection, and pharmaceutical agent application requires high cost. Further studies are warranted to optimize the procedures for neo-lymphangiogenesis induction by a simple method.

Limitations of the study included the small number of the cases and without histological confirmation of new lymphatic pathways. It would be ideal to confirm induction of neolymphangiogenesis histologically but it is ethically difficult to take biopsies after the treatment and this could also theoretically impact the new lymphatic channels. According to previous reports of close relationship between ICG lymphography findings and histologic findings, it is reasonable to evaluate creation of new lymphatic pathways solely based on ICG lymphography findings $(16,17,38)$. When a new linear pattern is seen on ICG lymphography after SDN, it can be considered evidence of a new lymphatic pathway $(19,39,40)$. This study failed to show statistically significant volume reduction after SDN procedure, although the postoperative volume showed a tendency to reduction. To confirm efficacy of SDN to improve lymphedema management, further prospective studies with larger cohort are warranted.

\section{CONCLUSIONS}

Subdermal dissection, although its probability is not high, has a potential to induce neo-lymphangiogenesis. Further studies are required to test and improve efficacy of the procedure for new lymphatic pathway creation.

\section{CONFLICT OF INTEREST AND}

\section{DISCLOSURE}

The authors declare no competing financial interests exist.

\section{REFERENCES}

1. Chang, DW, J Masia, R Garza 3rd, et al: Lymphedema: Surgical and medical therapy. Plast. Reconstr. Surg. 138 (2016), 209S-218S.

2. Chen, WF, M McNurlen, J Ding, M Bowen: Vascularized lymph vessel transfer for extremity lymphedema - Is transfer of lymph node still necessary? Int. Microsurg. J. 3 (2019), 1. DOI: 10.24983/scitemed.imj.2019.00119

3. Yamamoto, T, N Yamamoto, K Doi, et al: Indocyanine green (ICG)-enhanced lymphography for upper extremity lymphedema: A novel severity staging system using dermal backflow (DB) patterns. Plast. Reconstr. Surg. 128 (2011), 941-947.

4. Yamamoto, T, N Yamamoto, H Sakai, et al: Lymphedema quality of life score (LeQOLiS): A simple method for evaluation of subjective symptoms in extremity lymphedema patients. Plast. Reconstr. Surg. 2018 Aug 14 [epub ahead of print].

5. Yamamoto, T: Impact of lower extremity dysmorphia on lymphedema patients' quality of life. Plast. Reconstr. Surg. 2019 Feb 1 [epub ahead of print]

6. Mehrara, BJ, JC Zampell, H Suami, DW Chang: Surgical management of lymphedema: Past, present, and future. Lymphat. Res. Biol. 9 (2011), 159-167.

7. Yamamoto, T, N Yamamoto, T Numahata, et al: Navigation lymphatic supermicrosurgery for the treatment of cancer-related peripheral lymphedema. Vasc. Endovasc. Surg. 48 (2014), 139-143.

8. Executive Committee of the International Society of Lymphology. The diagnosis and treatment of peripheral lymphedema: 2020 Consensus Document of the International Society of Lymphology. Lymphology. 53 (2020), 3-19.

9. Yamamoto, T, M Narushima, K Kikuchi, et al: Lambda-shaped anastomosis with intravascular stenting method for safe and effective lymphaticovenular anastomosis. Plast. Reconstr. Surg. 127 (2011), 1987-1992.

10. Chang, DW, H Suami, R Skoracki: A prospective analysis of 100 consecutive 
lymphovenous bypass cases for treatment of extremity lymphedema. Plast. Reconstr. Surg. 132 (2013), 1305-1314.

11. Yamamoto, T, M Narushima, H Yoshimatsu, et al: Minimally invasive lymphatic supermicrosurgery (MILS): Indocyanine green lymphography-guided simultaneous multi-site lymphaticovenular anastomoses via millimeter skin incisions. Ann Plast. Surg. 72 (2014), 67-70.

12. Cheng, MH, TT Liu: Lymphedema microsurgery improved outcomes of pediatric primary extremity lymphedema. Microsurgery 40 (2020), 766-775.

13. Dayan, JH, CL Ly, RP Kataru, BJ Mehrara: Lymphedema: Pathogenesis and novel therapies. Annu. Rev. Med. 69 (2018), 263276.

14. Brorson, $\mathrm{H}$ : Liposuction in lymphedema treatment. J. Reconstr. Microsurg. 32 (2016), 56-65.

15. Granoff, MD, AR Johnson, K Shillue, et al: A single institution multi-disciplinary approach to power-assisted liposuction for the management of lymphedema. Ann. Surg. 2020 Nov 4. doi:

10.1097/SLA.0000000000004588. Epub ahead of print. PMID: 33156069.

16. Yamamoto, T, N Yamamoto, H Yoshimatsu, et al: Factors associated with lymphosclerosis: an analysis on 962 lymphatic vessels. Plast. Reconstr. Surg. 140 (2017), 734-741.

17. Yamamoto, T, M Narushima, I Koshima: Lymphatic vessel diameter in female pelvic cancer-related lower extremity lymphedematous limbs. J. Surg. Oncol. 117 (2018), 1157-1163.

18. Yamamoto, T, N Yamamoto, H Yoshimatsu, et al: Factors associated with lower extremity dysmorphia caused by lower extremity lymphedema. Eur. J. Vasc. Endovasc. Surg. 54 (2017), 126.

19. Yamamoto, T, N Yamamoto, Y Fuse, et al: Optimal sites for supermicrosurgical lymphaticovenular anastomosis: An analysis of lymphatic vessel detection rates on 840 surgical fields in lower extremity lymphedema. Plast. Reconstr. Surg. 142 (2018), 924e-930e.

20. Yamamoto, T: Onco-reconstructive supermicrosurgery. Eur. J. Surg. Oncol. 45 (2019), 1146-1151.

21. Yamamoto, T, N Yamamoto, T Kageyama, et al: Technical pearls in lymphatic supermicrosurgery. Global Hlth. Med. 2
(2020), 29-32.

22. Akita, S, N Mitsukawa, M Kuriyama, et al: Comparison of vascularized supraclavicular lymph node transfer and lymphaticovenular anastomosis for advanced stage lower extremity lymphedema. Ann. Plast. Surg. 74 (2015), 573-579.

23. Vignes, S, M Blanchard, A Yannoutsos, M Arrault: Complications of autologous lymphnode transplantation for limb lymphoedema. Eur. J. Vasc. Endovasc. Surg. 45 (2013), 516520.

24. Pons, G, J Masia, P Loschi, et al: A case of donor-site lymphoedema after lymph nodesuperficial circumflex iliac artery perforator flap transfer. J. Plast. Reconstr. Aesthet. Surg. 67 (2014), 119-123.

25. Telinius, N, VE Hjortdal: Role of the lymphatic vasculature in cardiovascular medicine. Heart 105 (2019), 1777-1784.

26. Rochlin, DH, S Inchauste, J Zelones, DH Nguyen: The role of adjunct nanofibrillar collagen scaffold implantation in the surgical management of secondary lymphedema: Review of the literature and summary of initial pilot studies. J. Surg. Oncol. 121 (2020), 121-128.

27. Forte, AJ, D Boczar, MT Huayllani, et al: Use of vascular endothelial growth factor-d as a targeted therapy in lymphedema treatment: A comprehensive literature review. Lymphat. Res. Biol. (2021) Mar 19. doi: 10.1089//rb.2020.0011. Epub ahead of print.

28. Yamamoto, T, M Narushima, K Doi, et al: Characteristic indocyanine green lymphography findings in lower extremity lymphedema: The generation of a novel lymphedema severity staging system using dermal backflow patterns. Plast. Reconstr. Surg. 127 (2011), 1979-1986.

29. Yamamoto, T, N Matsuda, K Doi, et al: The earliest finding of indocyanine green (ICG) lymphography in asymptomatic limbs of lower extremity lymphedema patients secondary to cancer treatment: The modified dermal backflow (DB) stage and concept of subclinical lymphedema. Plast. Reconstr. Surg, 128 (2011), 314e-321e.

30. Yamamoto, T, M Narushima, H Yoshimatsu, et al: Indocyanine green velocity: Lymph transportation capacity deterioration with progression of lymphedema. Ann. Plast. Surg. 71 (2013), 591-594.

31. Yamamoto, T, N Yamamoto, H Yoshimatsu, et al: Indocyanine green lymphography for 
evaluation of genital lymphedema in secondary lower extremity lymphedema patients. J. Vasc. Surg, Venous Lymphat, Disord. 1 (2013), 400-405.

32. Yamamoto, T, H Yoshimatsu, M Narushima, et al: Indocyanine green lymphography findings in primary leg lymphedema. Eur. J. Vasc. Endovasc. Surg. 49 (2015), 95-102.

33. Yamamoto, T, N Matsuda, T Todokoro, et al: Lower extremity lymphedema index: a simple method for severity evaluation of lower extremity lymphedema. Ann. Plast. Surg. 67 (2011), 637-640.

34. Yamamoto, T, N Yamamoto, N Hayashi, et al: Practicality of lower extremity lymphedema index: Lymphedema index versus volumetry-based evaluations for bodytype corrected lower extremity volume evaluation. Ann. Plast. Surg. (2016) Jan 30 [epub ahead of print]

35. Akita, S, N Mitsukawa, T Kazama, et al: Comparison of lymphoscintigraphy and indocyanine green lymphography for the diagnosis of extremity lymphoedema. J. Plast. Reconstr. Aesthet. Surg. 66 (2013), 792-798.

36. Yamamoto, T, T Iida, N Matsuda, et al: Indocyanine green (ICG)-enhanced lymphography for evaluation of facial lymphoedema. J. Plast. Reconstr, Aesthet, Surg. 64 (2011), 1541-1544.

37. Yamamoto, N: Lymphography change after liposuction: Possible neo-lymphangiogenesis by surgical trauma. J. Plast. Reconstr. Aesthet. Surg. (2021), Jun 3 [Epub ahead of print]
38. Aksoyler, D, O Bitik, FD Menku Ozdemir, et al: A new experimental lymphedema model: Reevaluating the efficacy of rat models and their clinical translation for chronic lymphedema studies. Ann Plast Surg. (2020) Jul 21. doi: 10.1097/SAP.0000000000002479. Epub ahead of print.

39. Yamamoto, T, T Iida, H Yoshimatsu, et al: Lymph flow restoration after tissue replantation and transfer: Importance of lymph axiality and possibility of lymph flow reconstruction using free flap transfer without lymph node or supermicrosurgical lymphatic anastomosis. Plast. Reconstr. Surg. 142 (2018), 796-804

40. Yamamoto, T, H Yoshimatsu, N Yamamoto: Complete lymph flow reconstruction: A free vascularized lymph node true perforator flap transfer with efferent lymphaticolymphatic anastomosis. J. Plast. Reconstr. Aesthet. Surg. 69 (2016), 1227-1233.

Takumi Yamamoto, MD, PhD

Department of Plastic and Reconstructive Surgery

Center Hospital of National Center for Global

Health and Medicine

1-21-1 Toyama, Shinjuku-ku,

Tokyo, Japan 162-8655

Tel.: +8133202 7181

Fax: +81332071038

E-mail: tyamamoto-tky@umin.ac.jp 